Commun:

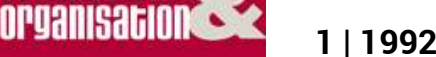

Communication et sentiment d'appartenance

\title{
Popularité du maire comme phénomène ethnique
}

Jean Tournon

\section{OpenEdition}

Journals

Édition électronique

URL : http://journals.openedition.org/communicationorganisation/1556

DOI : 10.4000/communicationorganisation. 1556

ISSN : 1775-3546

Éditeur

Presses universitaires de Bordeaux

Édition imprimée

Date de publication : 1 mai 1992

ISSN : 1168-5549

\section{Référence électronique}

Jean Tournon, «Popularité du maire comme phénomène ethnique », Communication et organisation

[En ligne], 1 | 1992, mis en ligne le 26 mars 2012, consulté le 19 avril 2019. URL : http://

journals.openedition.org/communicationorganisation/1556 ; DOI : 10.4000/

communicationorganisation. 1556

Ce document a été généré automatiquement le 19 avril 2019

(c) Presses universitaires de Bordeaux 


\title{
Popularité du maire comme phénomène ethnique
}

\author{
Jean Tournon
}

1 Le point de départ de mon analyse pourrait-être l'extraordinaire discordance dans l'opinion publique française entre la popularité des maires et l'impopularité des hommes politiques. Les sondages indiquent à peu près, et depuis des années, que 3/4 des français environ disent qu'ils ont confiance dans les maires en général, et que seulement un quart disent qu'ils ont confiance dans les hommes politiques comme si les maires n'étaient pas des hommes politiques. L'essai que je vous propose ce matin veut donner raison aux français qui voient dans les maires des hommes publics pas comme les autres. J'y développe l'hypothèse que le champ municipal soumet les hommes politiques à une forte sollicitation qui n'est pas vraiment démocratique, que j'appelle ethnique car, bien avant que l'État ne s'occupe d'elle et selon un faisceau de facteurs à la fois culturels, sociologiques et même politico-administratifs, la commune constitue une collectivité humaine assimilable à un groupe ethnique et qui impose son modèle cohésif à ses élus. Le maire donc, je vais le décrire, dans un premier temps, comme étant à la tête de ce groupe ethnique et dans un deuxième temps j'examinerai les problèmes que cela peut poser du point de vue de la démocratie car la logique ethnique et la logique démocratique sont deux choses très différentes.

2 Le maire à la tête du groupe ethnique est un représenteur et un meneur. En tant que représenteur il joue un rôle unificateur et un rôle identificateur. Le maire de par sa position institutionnelle, unifie sa commune, marque son unité et apparaît lui-même comme le symbole de cette unité. En effet la république a récupéré du grand dessein monarchique l'idée de faire du maire un quasi-fonctionnaire. Le maire est un administrateur, entre autres choses, et à ce titre-là d'emblée il est forcément l'homme de toute la collectivité. C'est lui qui fait tous les mariages, c'est lui qui examine les problèmes, par exemple les demandes de permis de construire, de tout le monde; il a la responsabilité des biens de la commune et vous savez quel peut-être le symbolisme fortement unitaire des biens communaux tels que l'église ou l'école ou, dans certains cas, de monuments qui sont un legs du passé et qu'il s'agit de maintenir en bon état pour 
l'avenir. L'école, c'est là où la nouvelle génération du groupe ethnique va se former pour continuer le groupe. Donc, comme le dit un anthropologue politique, Marc Abeles, le maire incarne l'unité politique de la commune ; il en est le centre, c'est l'État qui l'y a mis. Mais il a aussi une position sociale centrale. Toutes les études qui sont faites sur la vie concrète des maires, montrent que les maires sont extrêmement sollicités. Non seulement pour faire des choses qui sont liées à leur fonction d'administrateur mais aussi pour participer à bien des phases de la vie sociale: aux obsèques, aux fêtes, à toutes les cérémonies, à tous les banquets ; leur présence est attendue presque partout. Tout ce qui se fait d'important, sur n'importe quel plan, dans la commune, à un moment donné va passer par la mairie, que ce soit obligatoirement parce qu'il faut aller dire au maire qu'il y aura des problèmes d'eau, de construction, de ceci, de cela, mais aussi, spontanément, parce que l'on sait bien que le maire est ou doit être au courant.

Donc le maire se trouve tangent à tous les groupes, les organisations, les réseaux de la commune; et ainsi, au minimum, il en est le point de contact et il les relie. En général, il fait plus que cela : il aide ces divers groupes à se connaître, à s'ajuster les uns aux autres et il peut aider (c'est là son rôle d'unificateur) à la solution des conflits entre eux ; il se sent fortement poussé à le faire, s'il voit que ces conflits affaiblissent la commune et, bien sûr, le maire va aussi essayer de provoquer des synergies, d'unifier les efforts des gens de sa commune, pour, tous ensemble avoir de meilleures chances de succès. Un des aspects de ce rôle unificateur, qui est peut-être celui qui est le plus symboliquement intéressant pour nous, c'est que le maire est toujours le combattant au premier rang de tout ce qui peut désagréger ou risquer de faire mourir la commune. On vient de voir qu'il œuvre à la solution, à la pacification des conflits internes, il est aussi celui qui va exprimer le vouloir vivre du groupe, qui va être son agent d'adaptation pour montrer qu'il n'est pas condamné, qu'il sera capable de passer à travers les nouvelles difficultés qui surgissent continuellement. N'oublions pas que la commune est toujours quelque chose de très petit par rapport à un environnement politico-administratif (de l'État) ou surtout un environnement économique assez effrayant que même les États aujourd'hui, dans une période d'internationalisation, ne peuvent plus maîtriser.

Crucialement le maire est le protecteur de l'identité du groupe, c'est-à-dire celui qui est responsable de sa survie comme collectivité historique et cela va se traduire par le fait qu'il est en quelque sorte mis en devoir de veiller à ce que le groupe ethnique ne perde pas son âme. Donc autant que l'unité sur laquelle il veille en protégeant et, si possible en enrichissant le lien social, c'est l'unicité du groupe dont le maire se trouve investi défenseur. D'ailleurs, ces deux concepts ne sont pas affectivement séparables. Il n'y a solidarisation autour de la commune que parce c'est elle, bien particulièrement, avec son propre nom, avec son histoire, avec sa physionomie paysagère et bâtie, et avec un ensemble bien particulier des concitoyens qui y évoluent. La commune ce n'est donc pas n'importe quels hectares et n'importe quels habitants qui seraient pris en nombre égal, ailleurs ou un peu plus loin. Contrairement à ce que des professionnels de la communication un peu présomptueux pourraient croire, le maire n'invente pas et eux ne fabriquent pas une identité au groupe ethno-territorial: tout au plus, dans la masse incommensurable des relations identitaires touchant ce territoire-là et ceux qui $\mathrm{y}$ habitent, peuvent-ils choisir d'en souligner ou d'en maquiller quelques-unes. L'ensemble des relations identitaires constitue une signature que chacun déchiffre partiellement et, sans doute, différemment de son voisin, du voyageur ou du chargé d'études; et aussi chacun le déchiffre différemment selon les moments; mais en fait c'est bien une 
signature, que chacun reconnaît et dans laquelle tous se reconnaissent peu ou prou, et à laquelle il importe que le maire fasse honneur. Le phénomène global de la relation du maire à l'identité-singularité du groupe ethno-territorial n'est pas qu'il la façonne mais au contraire qu'elle le façonne. Ce n'est pas qu'il la fait plier mais que lui s'y plie. Dire cela ce n'est pas nier sa capacité éventuelle à y apporter des retouches, voire une inflexion, c'est tout simplement rappeler à l'aide de faits bien connus, les mécanismes de soumission dont la commune, le groupe ethno-territorial dispose envers le maire. D'abord, le groupe prend généralement des garanties en exigeant de lui qu'il soit du pays. L'autochtonie, même si elle est peu strictement conçue et encore moins vérifiée, rassure sur une probabilité assez élevée de bonne intériorisation, en partie déjà faite mais aussi en partie à parfaire jour après jour, des valeurs singulières de la localité. L'exigence d'attaches locales, venant du plus grand nombre et pas seulement de ceux qui pourraient vouloir se réserver le poste, démontre la conscience qu'a le groupe d'être unique.

C'est parce qu'il n'est pas un interchangeable n'importe quoi qu'il ne conçoit pas d'être gouverné ou représenté par un interchangeable n'importe qui. À défaut d'autochtonie, à défaut d'un enfant du pays, il va lui demander des gages d'intégration et de conformisation. « Un élu du sol, c'est un individu qui doit jouer la carte de l'enracinement pour acquérir une légimité véritable. Il est jugé (je cite ici un collègue bordelais) sur son aptitude personnelle à s'intégrer à la culture locale ». Tout au long de son parcours il est ainsi sous amicale surveillance; la possible sanction électorale, lointaine et problématique, est infiniment moins efficace que celle immédiate de coopération ou de recroquevillement, de refus de coopération, qu'il rencontre cent fois par jour de la part des gens du pays auxquels il a à faire et dont l'appui lui est indispensable. Ainsi se produit, dit Abelès, comme un phénomène d'osmose entre l'élu et l'esprit de sa ville. Mabileau parle de la naturalisation du maire dans le milieu local, milieu très sensible, je cite encore un anthrophologue, à la faute de goût ou à la dissonnance. Cette identification à une identité collective spécifique du lieu n'est pas la fin des obligations du maire. On attend de lui qu'il active et qu'il renforce cette identité. En premier lieu, le maire est instauré gardien de la mémoire du groupe. Voyez la revue "Empreintes Mémoire d'hier et mémoire de demain » : il est question du Grand Théâtre de Bordeaux et dès que vous ouvrez l'éditorial, il y a la silhouette de Jacques Chaban-Delmas et un texte de lui. En mettant en place des moyens de sauvegarde des témoignages du passé, Bordeaux et les communes de l'agglomération bordelaise accomplissent une action essentielle pour la défense de leur patrimoine et de leur histoire. Le travail quotidien du maire c'est d'évoquer cette mémoire, ce passé, de magnifier les grands personnages, les habitudes, de montrer le prix qu'il attache au maintien du patrimoine, architectural ou autre. Mais c'est aussi de veiller à la défense de cette singularité, de ces particularismes dans le présent et là, je pourrais citer des quantités de textes plus ou moins drôlatiques sur les exigences de la fonction du maire selon qu'il est maire de la ville de Camembert, de Roquefort, de Bordeaux, etc. où il y a, évidemment, un certain éventail de connaissances très spécialisées qu'il est censé acquérir. En plus de cela on attend du Maire qu'il aide à maintenir les usages, festifs notamment. Par ailleurs le Maire active et promeut cette identité de la ville à l'extérieur, au-delà de ses frontières; on voit que la position personnelle du maire sur la scène nationale devient de cette manière-là un atout pour la ville. Dans le livre d'Abelès, il est montré comment le positionnement (qui aurait pu être source de grandes difficultés locales) du Maire qui passe de l'opposition à la majorité, devient au contraire un atout pour la ville qui sent un petit peu qu'elle devient un paradigme de la modernisation politique ou de l'ouverture politique en France. Enfin le Maire, comme je le disais, est 
chargé de projeter la ville dans l'avenir c'est-à-dire de montrer que les élus ne vont pas être pris au dépourvu par toutes les évolutions qui se dessinent et peuvent affecter la ville mais qu'au contraire, ils tiennent bien les choses en main, qu'ils vont assurer le devenir de la ville et que, grâce à eux, elle va perdurer. On a ainsi une identification de l'homme à la ville et de la ville à l'homme. Georges dit que le maire est un moyen d'identification de la communauté, Abelès dit qu'il existe comme incarnation du groupe, qu'il matérialise la permanence d'une appartenance commune. On a ainsi, en quelque sorte, un mariage un peu étonnant entre deux singularités qui s'épousent et qui s'épaulent; il y a même, dans un certain nombre de cas, un début de processus d'héroïsation ou de sacralisation; ce qui est tout à fait explicable car, en grandissant son leader représenteur, le groupe se grandit lui-même.

Mais la deuxième tâche que je vais évoquer après celle d'avoir unifié et porté l'identité du groupe c'est la tâche d'être le meneur du groupe. Il est son porte drapeau, je l'ai montré, je vais insister maintenant sur le fait qu'il en est le chef de guerre, qu'il n'est jamais plus beau que lorsque le groupe est agressé. Le maire prend une inportance exceptionnelle chaque fois que la commune est dans la tourmente. Il y a les situations de catastrophe, évidemment il y a les situations de guerre. J'ai trouvé beaucoup de choses sur le rôle éminent des maires pendant la guerre; je reviens sur le cas de la ville d'Auxerre parce qu'il est particulièrement clair. Pendant la $2^{\mathrm{e}}$ guerre mondiale, Auxerre avait un maire qui, voyant que les choses devenaient assez difficiles, je crois en 1941, a démissionné en invoquant des convenances personnelles. Il a été remplacé par un maire qui a fait ce qu'il a pu pendant la période de Vichy et qui évidemment, au moment de la libération, s'est retrouvé quelque peu inquiété, et en tout cas déclaré inéligible. L'opinion de la ville d'Auxerre a été très forte, elle en a voulu au maire qui avait démissionné et elle a plébiscité ce maire déclaré inéligible, qui s'est néanmoins présenté et dont la liste a obtenu un succès éclatant et l'a réélu maire. Le conseil d'État l'a cassé, je ne sais pas si le terme est exact, il a dû être remplacé, mais la ville avait ainsi nettement marqué qu'un maire qui l'avait accompagnée dans les moments difficiles avait sa reconnaissance. Cette condamnation en quelque sorte infamante, qu'elle refusait était vue comme une façon qu'avaient les parisiens, le pouvoir central de se mêler des affaires de la ville ; les gens ont fait front de manière quasiment unanime derrière ce maire.

7 Le maire est à l'avant dès qu'il peut identifier ou que la commune identifie pour lui des intérêts hostiles. Cela peut être les spéculateurs fonciers, les promoteurs, cela peut être des immigrants, l'agression peut aussi venir de la politique nationale, avec toutes sortes de grands projets (le TGV tout récemment) ou des dictates législatifs qui vont venir porter atteinte à une activité particulière de la commune; il y a aussi les décisions internationales, européennes de plus en plus souvent, contre lesquelles des maires vont se rebeller. On voit que, dans tous ces cas, les maires qui n'ont pas été disciplinés ont été soutenus par la ville qui se considère comme agressée et réagit en étant solidaire derrière son maire. Il y a un agresseur que toutes les études montrent être l'agresseur idéal parce qu'il est complètement impersonnel, lointain, extérieur à la collectivité, c'est l'État. Et un chercheur bordelais, Jean-Louis Marie, a montré que le maire est le combattant suprême contre l'État qui paralyse en imposant des charges et des règlementations. Si quelque chose n'est pas possible c'est à cause de l'État, si quelque chose est réalisé c'est malgré l'État. Ainsi le Maire est le chef, le champion du groupe ethno-territorial ; et, en retour, il est vivement soutenu par celui-ci lorsqu'il est dans l'adversité. 
8 Il y a un chapitre que je n'ai pas besoin de développer, c'est celui du maire général en chef de la lutte pour le développement économique : on voit bien aujourd'hui que le fait de se mettre à la tête de la lutte pour les emplois, pour la vitalité économique de la commune, fait du maire un personnage tout à fait semblable au chef du groupe ethnique qui conduisait son groupe jadis sur les chemins de la chasse ou de la guerre.

Le chef du groupe ethnique local que j'appellerai le maire ethnique, est en situation difficile par rapport à la logique démocratique parce que la logique du pouvoir autochtone c'est de faire prévaloir la cohésion alors que la logique de la démocratie c'est de faire prévaloir la majorité. J'ai montré, combien le groupe avait besoin d'un unificateur et d'un pacificateur; au contraire la décision démocratique exige de visibiliser et d'accentuer des clivages qui, sans être completement exogènes, ont été faconnés principalement à l'extérieur du groupe ethno-territorial. La lutte électorale exacerbe ces tensions, qui entre les élections, prennent un caractère officiel et permanent dans l'opposition, un outil indispensable pour l'alternance démocratique. Quelles sont les solutions que les communes trouvent à ce dilemme? La première solution est que l'élection est généralement vue comme une parenthèse, c'est une sorte de mauvais moment à passer pour le groupe ethnique, révélateur d'un certain nombre de divisions et de tensions. Mais les textes de nombreux auteurs montrent bien que, comme le dit l'un, une fois le rite de l'élection accompli, l'identité du maire se restructure différemment. Albert Mabileau, le grand spécialiste bordelais, parle de «l'étiquette que le candidat emprunte le temps d'une élection pour l'oublier sitôt qu'il est entré ou revenu sur la scène politique locale ». On a souvent fait l'analyse de la déception des militants qui pensaient avoir gagné une victoire et qui découvrent que leur maire n'est plus le chef de leur camp, de leur parti, mais qu'il est devenu le chef du groupe tout entier.

Il y a un certain nombre de solutions qui varient, selon que l'affirmation ethnique, d'un côté, et l'affirmation des clivages idéologiques nationaux, de l'autre côté, sont plus ou moins forts. On peut tracer deux axes, l'axe de l'affirmation ethnique et l'axe des clivages idéologiques : chaque commune se situe à un point particulier de la rencontre de ces deux axes. S'il y a une force égale de cette affirmation ethnique et de cette affirmation idéologique on a une ligne qui s'en va en diagonale dans ce tableau et qui est la ligne de probabilité de l'apolitisme. Sur cette ligne, vont se trouver une diversité de figures de l'apolitisme, un certain nombre de maires, y compris de grands maires qui sont arrivés pendant un temps plus ou moins long et quelquefois durablement, à rester en dehors des appartenances partisanes. En fonction de la plus ou moins forte affirmation ethnique et de la plus ou moins forte affirmation des clivages majorité-minorité, on voit aussi quelles sont les probabilités de parachutage dans la commune et les probabilités d'occurence de divers styles de gouvernement municipal. Avec la probabilité d'apolitisme sur cet axe en diagonale, c'est là aussi que l'on va trouver une personnalisation très poussée, le personnage n'étant pas forcément flamboyant : les grands maires apolitiques sont, si l'on reprend la dichotomie de Machiavel, plus souvent des renards que des lions. Ils sont les spécialistes très habiles de la formule d'entente des divers intérêts de la collectivité. Au contraire, si vous avez une affirmation ethnique très forte et des clivages idéologiques faibles dans la ville, il y a une emprise plus probable du pluralisme social local et donc de coalitions très vastes qui ne tiennent pas du tout compte des frontières partisanes.

11 En guise de conclusion, je voudrais faire deux observations. Premièrement il n'y a pas, contrairement à ce que beaucoup de politologues disent, une tendance à la nationalisation et à la politisation de la vie politique municipale en France. Il y a 
aujourd'hui une conjoncture qui, globalement, si on la compare sur plusieurs décennies et à plus forte raison si on remonte les siècles, est favorable à l'affirmation du maire ethnique plutôt qu'à l'affirmation du maire partisan idéologique. Les Bordelais ne me démentiront pas mais tout le Sud-Est de la France est encore plus probant. Ma deuxième remarque c'est que le maire ethnique n'est pas forcément le parangon ou la forme achevée de l'affirmation de soi du groupe ethno-territorial. Il faut se rappeler que l'institution du maire ou de la prédominance mayorale est assez largement l'œuvre du pouvoir central elle apparait plutôt comme une transposition locale de la figure monarchique, puis napoléoniènne, puis républicaine de l'autorité que comme une création ethno-territonale locale. Même si ensuite cette institution du maire a été très bien récupérée par la commune. Si on regarde les débuts de l'affirmation du groupe ethno-territorial, ce que Augustin Thierry a appelé la révolution municipale en France entre le $\mathrm{XI}^{\mathrm{e}}$ et le $\mathrm{xIII}^{\mathrm{e}}$ siècle, on voit que, dans la mesure où elle était une véritable conquête du pouvoir autochtone, elle n'a pas imposé une formule uniforme de gouvernement local mais il semble tout de même qu'elle a eu une sorte de prédilection pour les formes d'autorité collégiale. Il y avait souvent trois, quatre et jusqu'à une douzaine de consuls ou échevins qui étaient sur un pied d'égalité, et cette formule collégiale s'est avérée capable d'affirmer avec la dignité, l'efficacité et les pondérations désirables, l'unité et la volonté d'autonomie du groupe ethno-territorial local, sans faire courir les risques d'une concentration du pouvoir sur un seul homme. Si l'actuelle montée en puissance et en autonomie des collectifs ethno-urbains se confirmait et ne se heurtait pas à des modèles nationaux, trop monarchistes-présidentialistes d'exercice du pouvoir, peut-être qu'un certain retour à la collégialité, collégialité aussi bien compatible avec des formules de domination majoritaire qu'avec des formules de partage du pouvoir à la suisse ou à l'italienne, permettrait d'échapper aux tensions actuelles entre logique ethnoterritoriale et logique de la démocratie pluraliste.

\section{RÉSUMÉS}

Le maire, porte-parole et pacificateur de sa commune, en est aussi, de plus en plus, le chef de guerre pour l'emploi et les investissements. Tout cela s'accorde mal avec la logique démocratique qui voudrait faire de lui le meneur d'une majorité.

\section{AUTEUR}

\section{JEAN TOURNON}

Jean Tournon, chercheur à la Fondation Nationale des Sciences Politiques et Vice Président du Comité « Ethnicité et Politique » de l'Association Internationale de Science Politique, travaille, à l'institut d'Études Politiques de Grenoble (CERAT) sur le thème "Groupes d'intérêt et démocratie ", s'intéressant plus particulièrement, dans la période récente, aux groupes ethniques ("Construction et déconstruction du groupe ethnique » International Political Science. 1989. vol $10 \mathrm{n}^{\circ} 331-348$ ) 